\title{
SR Research Square

\section{The impact of probiotics supplementation on gut microbiota after Helicobacter pylori eradication: a multicenter, open-label, randomised trial}

Bo Tang

Xinqiao Hospital

Li Tang

Xinqiao Hospital

Cheng Huang

Chongqing Iron and Steel Hospital

Chuan Tian

Nanchuan People's Hospital

Ling Chen

958 Hospital of PLA

Zhijun He

Chongqing Fifth Peoples' Hospital

Guodong Yang

North Sichuan Medical College

Luo Zuo

Chengdu Medical College

Guoce Zhao

Xinqiao Hospital

En Liu

Xinqiao Hospital

\section{Sumin Wang}

Xinqiao Hospital

Hui Lin

Xinqiao Hospital

Jialin He

Xinqiao Hospital

Shiming Yang ( $\square$ Yangshiming@tmmu.edu.cn )

Xinqiao Hospital, Third Military Medical University https://orcid.org/0000-0002-5911-8746 
Keywords: Helicobacter pylori, therapy, gut microbiota, probiotics

Posted Date: March 19th, 2020

DOI: https://doi.org/10.21203/rs.3.rs-18049/v1

License: (c) (1) This work is licensed under a Creative Commons Attribution 4.0 International License. Read Full License 


\section{Abstract}

Background: Helicobacter pylori (H. pylori) eradication therapy may lead to the perturbation of gut microbiota. The role of probiotics in gut microbiota during eradication therapy is still debated.

Design: This was a multicentre, open-label, randomised trial done at seven hospitals in China. 162 patients were enrolled, 79 patients were randomly divided into group A (bismuth quadruple therapy), and 83 patients were randomly subjected into group B (bismuth quadruple therapy supplemented with Medilac-S). Faecal samples were collected before treatment and 2 weeks, 4 weeks, 6 weeks, and 8 weeks after eradication therapy. Gut microbiota was analyzed by $16 \mathrm{~S}$ rRNA high-throughput sequencing. This trial is complete and registered with Chinese Clinical Trial Registry (Chictr.org.cn, ChiCTR1900022116).

Results: The eradication rates of group A and group B were $82.43 \%$ and $87.01 \%$, respectively $(P>0.05)$. Compared with baseline, alpha and beta diversity was significantly altered 2 weeks after eradication in both group $A$ and group $B$, which was restored at week 8 . There were no significant differences in alpha and beta diversity between the two groups. Bismuth quadruple therapy resulted in enrichment of some detrimental bacteria taxa such as Klebsiella and Streptococcus that were not recovered by week 8 . Probiotics supplementation could rapidly restore the taxa levels of Klebsiella and Streptococcus by week 4 after eradication, and increase the beneficial taxa of Bacillus and Lactobacillales. Functional analysis revealed that lipopolysaccharide biosynthesis and polymyxin resistance pathways were significantly enriched after eradication therapy, while probiotics supplementation mainly enriched the cofactors and vitamins metabolism pathways. Several detrimental taxa were identified to be correlated with features of older age, alcohol use and high BMI, while probiotics supplementation could effectively restore the adverse impact in patients with these characteristics.

Conclusion: Probiotics supplementation is beneficial for patients during $H$. pylori eradication, especially for patients with older age, alcohol drinking, and obesity, which might obtain the maximum benefits.

\section{Introduction}

H. pylori $(\mathrm{H}$. pylori) is a gram-negative bacterium that infects over half of the world population, and causes various gastric diseases including gastritis, chronic atrophic gastritis, peptic ulcers, gastric mucosa associated lymphoid tissue (MALT) lymphoma, and even gastric cancer [1, 2]. Therefore, H. pylori eradication therapy is recommended to reduce the recurrence of peptic ulcer disease and the incidence of gastric cancer [3, 4].

Recently, the regimen of $\mathrm{H}$. pylori eradication therapy includes dual therapy, triple therapy, and the bismuth-containing quadruple therapy [5]. Triple therapy remains the standard of care in the published international guidelines of the European Helicobacter and Microbiota Study Group in areas of low clarithromycin resistance [3]. However, the clarithromycin- or metronidazole-based triple therapy for $\mathrm{H}$. pylori infection is no longer recommended in China because of its high antibiotic resistance and low eradication efficacy. Bismuth quadruple therapy is recommended as the first-line eradication regimen in 
China [6]. No matter which regimens were used in eradication therapy, several concerns and barriers regarding the widespread use of the antibiotics and proton pump inhibitors (PPIs) were raised [7-11]. H. pylori eradication consists of PPIs and antibiotics can cause the disruption of gut microbiota, which is considered as a major contributing factor in pseudomembranous colitis associated with Clostridium difficile infection, diarrhea, or antibiotic resistance [12]. PPIs can alter gastrointestinal pH which might affect gut microbiota and the survival of enteric pathogens. Moreover, the administration of broadspectrum antibiotics can reduce microbiota diversity, disrupt the microbiota, and enrich the antibioticresistant strains [13]. Eradication therapy may induce the pathogenesis of various disorders through the changes and dysbiosis in gut microbiota. Recently, several studies have assessed the impact of H. pylori eradication on the gut microbiota. Shortly after the H. pylori eradication therapy, the bacterial diversity was significantly reduced $[8,14-16]$. Therefore, studying the effects of eradication therapy on the composition of the gut microbiota and exploring the potential strategy to maintain the microbiota homostasis is extremely important.

Probiotics are kinds of microbes that are beneficial for the host health. Previous studies used the certain probiotics during eradication therapy to decrease side effects, improve compliance, and thereby increase eradication rates [5]. A study conducted in Spain where 209 consecutive patients were prescribed eradication therapy and randomly received probiotics (Lactobacillus plantarum and Pediococcus acidilactici) or matching placebo showed that the eradication rates and side effects were observed to be similar [17]. While some other studies showed that probiotics improved the eradication rate and decreased the incidence of diarrhea, abdominal distension and constipation [18]. Therefore, the role of probiotics in eradication therapy is still debated. Additionally, previous studies mainly focused on the effect of probiotics on the eradication rate and side effects with the standard triple therapy, few studies have analyzed the influence of probiotics on the gut microbiota on a community-wide scale and the function of the gut microbiome in bismuth quadruple therapy. Especially, the optimal supplementation such as the species, duration, dosage, and the suitable population is extremely limited.

In this study, we aimed to investigate the effects of probiotics supplementation on the homostasis and functional potential of gut microbiota after bismuth quadruple therapy, and further explore the optimal use of probiotics for the suitable people to gain the most beneficial effects of probiotics intake.

\section{Materials And Methods}

\section{Patients and study design}

This multicenter, open-label, randomized clinical trial was performed at seven hospitals in China from March 2019 to November 2019. Inclusion criteria: patients aged between 18 and 60 years, at least two positive tests of rapid urease test and ${ }^{13} \mathrm{C}$-urea breath test $\left({ }^{13} \mathrm{C}\right.$-UBT $)$. Patients with any one of the following criteria were excluded from the study: history of gastrectomy, previous eradication therapy for H. pylori, peptic ulcer or other upper gastrointestinal lesions, gastrointestinal malignant tumor, contraindication or previous allergic reactions to the study drugs, severe concurrent diseases or 
malignancy, pregnant or lactating women, the use of antiacids or gastric mucosal protective drugs or antibiotics or probiotics in the past two weeks, and patients who could not give informed consent. Written informed consent was obtained from all patients before enrolment, and this trial was approved by the Institutional Review Board of each participating hospital. This trial was registered at Chinese Clinical Trial Registry (Chictr.org.cn, ChiCTR1900022116).

Based on the inclusion and exclusion criteria, a total of 162 patients were enrolled in this study. Eligible patients were randomly assigned to two groups (group A and group B). Patients in group A received 14day bismuth quadruple therapy (BQT) consisting of esomeprazole $20 \mathrm{mg}$, amoxicillin $1000 \mathrm{mg}$, furazolidone $100 \mathrm{mg}$, bismuth potassium citrate $220 \mathrm{mg}$, all given twice daily. This eradication therapy was given due to the low rates of antibiotic resistance to amoxicillin and furazolidone in southwest China. Patients in group B received the 14-day BQT therapy supplemented with probiotics (Medilac-S; Enterococcus faecium $4.5 \times 10^{8}$ and Bacillus subtilis $5.0 \times 10^{7}$, Hanmi, Beijing, China) three times a day for 4 weeks. Gastrointestinal symptoms were assessed at baseline for all patients and on week 2, week 4, week 6 , and week 8 after therapy. ${ }^{13} \mathrm{C}$-UBT was used to evaluate the $\mathrm{H}$. pylori eradication effect at 6 weeks after completion of treatment.

\section{Fecal sample collection}

Fresh stool samples were collected from all patients at baseline (before treatment), at week 2, week 4, week 6 , and week 8 . Participants were asked to return the faecal specimen to the research assistant in the hospital on the day of sample collection. All stool samples were immediately frozen and stored at $-80^{\circ} \mathrm{C}$.

\section{S rRNA gene amplification and sequencing}

Total DNA from the fecal samples was isolated from the TIANamp Stool DNA Kit (TIANGEN Biotech Co. Ltd., Beijing, China) according to the manufacturer's instructions. DNA concentration was quantified using a Nanodrop (Thermo Scientific, Wilmington, USA), and its integrity was assessed by $1 \%$ agarose gel electrophoresis. DNA was stored at $-20^{\circ} \mathrm{C}$ until use. The V3-V4 hypervariable regions of the $16 \mathrm{~S}$ rRNA were amplified using the following primers: forward Primer: 5'-ACTCCTACGGGAGGCAGCAG-3' and reverse primer 5'-GGACTACHVGGGTWTCTA AT-3'. The amplifications were performed employing a step cycling protocol consisting of $95^{\circ} \mathrm{C}$ for $30 \mathrm{~min}, 25$ cycles of $98{ }^{\circ} \mathrm{C}$ for $15 \mathrm{~s}, 55^{\circ} \mathrm{C}$ for $30 \mathrm{~s}$, and $72{ }^{\circ} \mathrm{C}$ for $45 \mathrm{~s}$, ending with the final elongation at $72^{\circ} \mathrm{C}$ for $10 \mathrm{~min}$. PCR amplicons were purified using Agencourt AMPure Beads (Beckman Coulter, Indianapolis, IN) and quantified using the PicoGreen dsDNA Assay Kit (Invitrogen, Carlsbad, CA, USA). The purified amplicons were then sequenced on an Illumina Miseq platform (Illumina, San Diego, USA) by Longsee Biomedical Corporation (Guangzhou, China).

\section{Sequence analysis}

The Quantitative Insights into Microbial Ecology 2 (QIIME2, version 2019. 7) platform in our Ubuntu Linux server was used to process the sequencing data. In the bioinformatics analysis, the fastq format sequence files (spanning the entire 16S rRNA gene V3-V4 region) were loaded into fastqc for basic quality control. Pair-end sequences that passed the basic quality control were then merged and denoised using 
"qiime dada2 denoise-paired" command of QIIME2 with parameters: $p$-trim-left-f $=9$, p-trim-left-r $=9, p$ trunc-len- $f=250$, $p$-trunc-len- $r=250$. DADA2 is a pipeline for detecting and correcting (where possible) Illumina amplicon sequence data [19]. DADA2 group unique sequences and referred to as sequence variants (the equivalent of $100 \%$ OTUs in QIIME 1) [19]. As implemented in the q2-dada2 plugin, this quality control process will additionally filter any phiX reads (commonly present in marker gene Illumina sequence data) that are identified in the sequencing data, and will filter chimeric sequences. The "qiime feature-table summarize" command was used to generate a feature-table listing how many sequences are associated with each sample and with each feature. The lowest sequences count of all the samples were used as the sampling-depth. Then the "qiime feature-table rarefy" command was used to subsample frequencies from all samples so that the sum of frequencies in each sample is equal to sampling-depth [20].

All the alpha diversity and beta diversity results were calculated by "qiime diversity alpha" and "qiime diversity beta" command from the subsampled feature-table (also called rarefied table). The PCoA results were calculated by "qiime diversity pcoa" command and visualized by "qiime emperor plot" command.

A pre-trained Naive Bayes classifier (silva-132-99-nb-classifier.qza) and the "qiime feature-classifier classify-sklearn" command were used to explore the taxonomic composition of the samples. This classifier was trained on the Silva database version 132 [21] with $99 \%$ similarity, where the sequences have been trimmed to only include the V3-V4 region of the $16 \mathrm{~S}$ that was sequenced in this analysis. The Wilcoxon tests were used to evaluate the ecological similarity between and within groups. Furthermore, linear discriminant analysis effect size (LEfSe) was used to select significant candidates at the genus level. We compare relative abundance of taxa between the two groups and different time period within each group using a nonparametric Mann-Whitney $U$ test, followed by a Linear Discriminant Analysis (LDA) to estimate the effect size of each microbial feature with differential abundance. It was considered as significantly enriched taxa with LDA score greater than 2.0 at a $P$ value $<0.01$.

\section{Functional pathway prediction}

The 16S rRNA functional prediction by used amplicon sequence variants (ASVs) is performed by PICRUSt2 (Phylogenetic Investigation of Communities by Reconstruction of Unobserved States, version 2). Then ASVs were categorized into Clusters of Orthologous Groups (COG) and into Kyoto Encyclopedia of Genes and Genome (KEGG) orthology (KO). According to the COG database, the descriptive information of each $\mathrm{COG}$ and its functional information were parsed from the eggNOG database to obtain the functional abundance spectrum. KO, Pathway and Enzyme (EC) information were obtained according to the KEGG database while the abundance of each functional category was calculated according to OTU abundance.

\section{Clinical correlation analysis}

To analyze the correlation between gut microbiota and clinical demographic variables of these selected patients, we employ the non-parametric multivariate analysis of variance (Adonis), an analysis utilizing a matrix of arbitrary squares computed pairwise distances in replace of the covariance matrix that are 
decomposed into within and between group sums of squares. This analysis was conducted employing the adonis function from the $R$ package vegan. After the significant variables were identified, the specific bacteria taxa were identified based on the different clinical features using LEfse analysis.

\section{Statistical analyses}

Data was presented as mean \pm standard deviation (SD). We used the $\chi 2$ test or Fisher's exact test for analysis of categorical data and Student's $t$ test or the ANOVA test for analysis of continuous data. Eradication efficacy was performed on an intention-to- treat (ITT) population where patients who dropped out were considered as treatment failures. Secondary per-protocol (PP) analyses were performed which excluded patients lost to follow-up or prematurely withdrew before completion of the study. Differences in relative abundance of bacteria taxa between group $A$ and group $B$ were compared by Wilcoxon rank sum test. All tests were performed using GraphPad Prism (v6.0) (GraphPad Software, Inc., CA, U.S.A.), SPSS Statistics (V. 24.0.0.0) (SPSS Inc., Chicago, U.S.A.) or R software (version 3.4.4). All statistical tests were two-tailed. P-values $<0.05$ were considered significant.

\section{Results}

\section{Baseline characteristics}

In this study, 162 patients were enrolled, 79 patients were randomly divided into group A, and 83 patients were randomly subjected into group B. 151 patients completed this trial, while 5 patients in group $A$ and 6 patients in group B withdrew from this study (Fig. 1). Finally, a total of 755 stool samples were collected and subjected to analysis. The baseline characteristics of enrolled patients are presented in Table 1. No significant differences in age, gender, body mass index (BMI), smoking habits, alcohol consumption, marital status, education and exercise were found between the two groups (all $P>0.05$ ). Before the eradication therapy, there was no significant difference in overall gastrointestinal symptoms between the two groups (Supplementary Table S1). Since the diet components had potential impact on the gut microbiota, we then assessed nutrient intake using a Food Frequency Questionnaire (FFQ) in these patients and found that there was no difference between the two groups (Supplementary Table S2). The eradication rates of group A and group B were $82.43 \%$ and $87.01 \%$ by ITT analysis. By PP analysis, the rates in group $A$ and group $B$ were $84.72 \%$ and $89.33 \%$, respectively. No significant differences in eradication rates were observed $(P>0.05)$ (Table 2$)$. 
Table 1

Baseline characteristics of patients in the two groups.

\begin{tabular}{|c|c|c|c|}
\hline \multicolumn{4}{|c|}{ Characteristics Group A $(n=74)$ Group B $(n=77) P$} \\
\hline Gender & & & 0.122 \\
\hline male & $44(59.46 \%)$ & $55(71.43 \%)$ & \\
\hline female & $30(40.54 \%)$ & $22(28.57 \%)$ & \\
\hline $\mathrm{Age}^{\mathrm{a}}$ & $45.32 \pm 10.98$ & $43.29 \pm 11.30$ & 0.263 \\
\hline $\mathrm{BMI}^{\mathrm{a}}$ & $23.30 \pm 3.48$ & $23.30 \pm 3.65$ & 0.996 \\
\hline Education & & & 0.904 \\
\hline primary & $15(20.27 \%)$ & $12(15.58 \%)$ & \\
\hline junior & $21(28.38 \%)$ & $22(28.57 \%)$ & \\
\hline senior & $20(27.03 \%)$ & $22(28.57 \%)$ & \\
\hline bachelor & $17(22.97 \%)$ & $18(23.38 \%)$ & \\
\hline postgraduate & $1(1.35 \%)$ & $3(3.90 \%)$ & \\
\hline Job & & & 0.607 \\
\hline Manual labor & $22(29.73 \%)$ & $20(25.97 \%)$ & \\
\hline Mental labor & 52 (70.27\%) & 57 (74.03\%) & \\
\hline Marital status & & & 0.219 \\
\hline unmarried & $9(12.16 \%)$ & $15(19.48 \%)$ & \\
\hline married & 65 (87.84\%) & $62(80.52 \%)$ & \\
\hline Smoking & $13(17.57 \%)$ & $12(15.58 \%)$ & 0.915 \\
\hline Alcohol & 17 (22.97\%) & $14(18.18 \%)$ & 0.676 \\
\hline Yogurt & $9(12.16 \%)$ & 11 (14.29\%) & 0.700 \\
\hline Exercise & $11(14.86 \%)$ & $14(18.18 \%)$ & 0.584 \\
\hline
\end{tabular}


Table 2

Efficacy of $\mathrm{H}$. pylori eradication in the two groups.

\begin{tabular}{llll}
\multicolumn{4}{l}{ Analysis Group A $(\mathbf{n}=\mathbf{7 4})$ Group B $(\mathbf{n}=\mathbf{7 7})$ P } \\
$\begin{array}{llll} \\
\text { PP } & 61 / 72(84.72 \%) & 67 / 75(89.33 \%) & 0.226 \\
\text { ITT } & 61 / 74(82.43 \%) & 67 / 77(87.01 \%) & 0.405\end{array}$
\end{tabular}

Group A: bismuth quadruple therapy; Group B: bismuth quadruple therapy + probiotics

supplementation with Medilac-S; ITT, intention to treat; PP, per protocol; Data are presented as n (\%).

\section{Alterations in gut microbial diversity after $H$. pylori eradication and probiotics supplementation}

We first evaluated the alpha diversity in each group using four indices chao 1, observed OTUs, Shannon and faith_pd index. The alpha diversity indices were significantly decreased 2 weeks after treatment in both groups $(P<0.01)$, which lasted for up to 4 weeks after treatment $(P<0.05)$. There was a trend for restoration of microbiota with time. The alpha diversity almost returned to the baseline at week 8 (Figure $2 \mathrm{~A}$, Supplementary Figure S1). As for the comparison between the two groups, there were no significant differences in alpha diversity at baseline, week 2, week 4, week 6 and week $8(P>0.01$; Figure 2B and C, Supplementary Figure S2).

In order to examine the variability of microbial community between the two groups, we calculated beta diversity using PCoA on weighted Uniface distance. There were no significant differences in the beta diversity between the two groups before treatment $(P=0.652$; Figure 2D). However, there were significant differences in the beta diversity at week 2, week 4, week 6 and week 8 after treatment compared to the baseline based on all patients or each group alone (Figure 2E and F). There were no significant differences in beta diversity between the two groups at each time period (Figure 2F).

\section{Changes in microbiota taxa after $H$. pylori eradication and probiotics supplementation}

In order to identify the profiles of gut microbiome changes, we examined the microbiota taxonomic composition and relative abundance in the two groups at different taxonomic levels. At the phylum level, the relative abundance of Proteobacteria was significantly increased, while the abundance of Firmicutes, Bacterioidetes, Verrucomicrobia, and Actinobacteria was significantly decreased 2 weeks after treatment in both groups (Figure 3A). No significant difference in phyla was observed at week 4, week 6 , and week 8 compared to the baseline (Figure 3A). Meanwhile, there was a decrease in the Bacterioidets:Firmicutes (B:F) ratio at week 2 after treatment which returned to the baseline from week 4 to week 8 (Figure 3B). At genus level, we observed an increased abundance of Clostridium, Klebsiella, Streptococcus, and 
Veillonella, while a decrease of Bacteroides, Faecalibacterium, Roseburia, Lachnospira, Phascolarctobacterium, Megamonas, Oscillospira, and Ruminococcus at 2 weeks after treatment compared to the baseline in both groups (Figure $3 \mathrm{C}$ and $\mathrm{D}$ ).

We further used LEfSe to specifically identify the bacterial taxa in each group at different periods after eradication. In group A, several bacterial taxa were differentially abundant compared to the baseline with linear discriminant analysis (LDA) score $>2$ and $\mathrm{P}<0.01$. Klebsiella, Streptococcus, Veillonella, Fusobacterium, Morganella, and Prevotella were significantly enriched, while Bacteroides, Faecalibacterium, Roseburia, Lachnospira, Phascolarctobacterium, Bifidobacterium, and Butyricimonas were markedly decreased at week 2 (Supplementary Figure S3A). With the prolonged time periods, we observed that some of these bacterial taxa changes were transient, such as the increase of Prevotella, Fusobacterium, and Morganella, together with a decrease in Bacteroides, Faecalibacterium, Lachnospira, Bifidobacterium, and Butyricimonas at week 2 which recovered to the baseline by week 4 . However, some other changes were longer lasting, such as the increase in relative abundance of Klebsiella,

Streptococcus, and Veillonella, as well as a decrease of Roseburia and Oscillospira at week 2 which did not return to baseline by week 8 (Supplementary Figure S3A-D).

Similar in group B, Klebsiella, Streptococcus, and Veillonella were also significantly enriched, while Bacteroides, Faecalibacterium, Roseburia, Lachnospira, Phascolarctobacterium, Sutterella, Akkermansia, and Bifidobacterium were markedly decreased at week 2 (Supplementary Figure S3E). Differently, Enterococcus and Bacillus, main components of the probiotics, were markedly enriched at week 2 and week 4, normalized by week 6 and week 8, indicating the colonization of the probiotics during its supplementation (Supplementary Figure S3E-H). Furthermore, the Klebsiella and Streptococcus rapidly returned to normal by week 4 after probiotics supplementation (Supplementary Figure S3E-H).

To specify the distinct microbial taxa between the two groups at each time point, we further performed LEfSe to compare bacterial abundances. We observed that Enterococcus, Citrobacter, and Oscillospira were significantly enriched in group B, while Dialister, Anaerotruncus, and Megasphaera were mainly enriched in group A at week 2 (Figure 4A). At week 4, Enterococcus, Bacillus, and Lactobacillales were enriched in group $B$ (Figure 4B), suggesting the successful colonization of probiotics after its supplementation. By week 6 and week 8, the enriched abundance of Enterococcus and Bacillus disappeared (Figure 4C and D). The above results suggested that $H$. pylori eradication could significantly disturb the composition of gut microbiota, enrich some detrimental bacteria taxa such as Klebsiella and Streptococcus, while decrease some beneficial taxa like Faecalibacterium, Roseburia, Lachnospira, Phascolarctobacterium, Bifidobacterium, and Butyricimonas. The effective colonization of probiotics could be observed during its supplementation, and the probiotics supplementation might rapidly decrease the enrichment of some detrimental bacteria taxa.

\section{Predictive functional pathways of microbial community after $H$. pylori eradication and probiotics supplementation}


To investigate the potential role of gut microbiome, we tried to identify the functional variations in the microbial communities using PICRUSt analysis with KEGG database to predict microbiota associated functional pathways. We observed differential predicted functions between samples before and after $H$. pylori treatment. At week 2 in group A, pathways involved in starch degradation, pyruvate fermentation, glycolysis, glucarate degradation were decreased, whereas those involved in fatty acid oxidation, sucrose degradation, formaldehyde assimilation were increased at week 2 (Supplementary Figure S4A). Consistent with the tendency of microbial changes, we observed some changes of the predicted pathways were also transient, while some other changes such as an increase of sucrose degradation and fatty acid oxidation as well as the decrease of starch degradation and glycolysis were longer lasting until week 8 (Supplementary Figure S4A-D). In group B, pathways involved in propanediol degradation, fatty acid oxidation, pyruvate_dehydrogenase, hexitol_fermentation sucrose_degradation, methylphosphonate_degradation were increased, whereas those involved in glycogen_degradation, peptidoglycan_biosynthesis, L_histidine_biosynthesis, coenzyme_A_biosynthesis,

formaldehyde_oxidation were decreased at week 2 (Supplementary Figure S4E). Similarly, most of these changes seemed to be transient, while some others were lasting until week 8 (Supplementary Figure S4EH).

To further compare the differential functional pathways between the two groups, we conducted LEfSe analysis to identify the discriminating functional pathways. Interestingly, the distinct pathways were only identified between the two groups at week 4 . We predicted 9 different microbiota-associated functional pathways between group A and group B. Four pathways with increased abundance in group B were predicted, including superpathway of thiamin diphosphate biosynthesis, sulfate_reduction, coenzyme_A_biosynthesis, and N10 formyl tetrahydrofolate biosynthesis. In contrast, the abundances of 5 pathways, which were associated with superpathway_of_lipopolysaccharide_biosynthesis, polymyxin_resistance, starch degradation, CDP_diacylglycerol_biosynthesis, and mannan_degradation were significantly decreased compared with group A (Figure 5A).

Furthermore, we investigated whether the differential microbial taxa between group A and group B were related to the predicted pathways to depict how specific taxa was involved in the functional pathways. The Enterococcus was significantly enriched in group B after probiotics supplementation, and we found Enterococcus was related to five of the nine predicted pathways at week 4. Specifically, Enterococcus was positively associated with superpathway of thiamin diphosphate biosynthesis, N10 formyl tetrahydrofolate biosynthesis, and coenzyme_A_biosynthesis, while negatively related to superpathway of lipopolysaccharide biosynthesis and polymyxin_resistance (Figure 5B-F). In terms of the Collinsella, which was significantly decreased in group $B$, was positively related to starch degradation and CDP_diacylglycerol_biosynthesis, and mannan_degradation (Figure 5G-I). Taken together, we hypothesized that the altered gut microbiota after eradication and probiotics supplementation might contribute to the various metabolic or clinical features through these microbiota related pathways.

\section{Gut microbiome correlated with patients' features}


Since various factors such as diet, age, alcohol, smoke, BMI might have impact on gut microbiota, we sought to investigate the relationship of gut microbiome and these factors after eradication. We incorporated the following variables into analysis: age, gender, BMl, alcohol, smoking, exercise. We found that age, alcohol and BMI were closely correlated with the microbial community diversity. We further identified specific bacterial taxa in group $A$ and group $B$ by dividing patients into different subgroups based on these factors. The analysis showed that Streptococcus, Dorea, Selenomonas, and Mogibacterium were significantly increased, while Paraprevotella and Turicibacter decreased in elder patients in group A (Figure 6A). However, Ruminococcus was significantly increased with a decrease of Megasphaera in elder patients in group B (Figure 6A). Compared to non-drinkers, Gemmiger and Collinsella were increase after eradication, while Sporosarcina, Alloscardovia, and Moryella were enriched after probiotics supplementation (Figure 6B). In terms of BMI, we found that Prevotella, Bilophila, Dialister, and Eikenella were markedly enriched after eradication, while Megasphaera, Blautia, and Faecalibacterium were increased after probiotics supplementation in obesity patients (Figure 6C). These results indicated that age, $\mathrm{BMI}$ and alcohol had an impact on gut microbiota after $\mathrm{H}$. pylori eradication, and probiotics supplementation might partially eliminate the adverse effect in patients with these specific features.

\section{Discussion}

In this study, we performed a multicenter, randomized trial to show the distinct effects of bismuth quadruple therapy and probiotics (Medilac-S) supplementation on gut microbiota. Various studies have shown that the administration of antibiotics reduces the diversity of the gut microbiota [14-16]. Alpha diversity decreased 1 week post-eradication therapy and was restored to almost pre-eradication levels 8 weeks later [22]. Consistent with these studies, our data also presented a significant disruption of gut microbiota at the end (week 2) of eradication therapy. We then observed a trend of gradual restoration with time from week 4 to week 8 , and the alpha and beta diversities were almost restored at week 8 in both groups. Unlike the previous study that probiotics could maintain the diversity of gut microbiota after eradication therapy [14], we found that probiotics supplementation failed to increase or maintain the diversity after $\mathrm{H}$. pylori eradication.

In accordance with previous reports [22, 23], in our current study, the gut microbiota before H. pylori eradication therapy predominantly contained commensal microbes of the phyla Firmicutes, Bacteroidetes, and Proteobacteria. Different eradication regimens containing different antibiotics might exert distinct effects on gut microbiota [15]. Oh, et al showed that the relative abundance of Firmicutes decreased and that of Proteobacteria increased immediately after triple therapy [14]. Hsu and colleagues showed that the relative abundance of Proteobacteria increased, whereas that of Bacteroidetes, Actinobacteria, and Verrucomicrobia decreased immediately after bismuth quadruple therapy containing pantoprazole, bismuth tripotassium dicitrate, tetracycline, and metronidazole [8]. Another study showed that reverse therapy containing pantoprazole, amoxicillin, clarithromycin, and metronidazole reduced the relative abundances of Firmicutes and Actinobacteria, while increased the abundance of Proteobacteria [24]. The enrolled patients in our present study received a 14-day bismuth quadruple therapy consisting of esomeprazole, amoxicillin, furazolidone, and bismuth potassium citrate. We observed that the relative 
abundance of Proteobacteria was significantly increased, while the abundance of Firmicutes, Bacterioidetes, Verrucomicrobia, and Actinobacteria was significantly decreased 2 weeks after treatment, which almost returned to the baseline levels at week 8 . Amoxicillin and clarithromycin was supposed to contribute to the reduction of Firmicutes and Actinobacteria following eradication [24]. Our data revealed that Bacterioidetes and Verrucomicrobia were also significantly reduced, which might be attributed to furazolidone. The dramatic increase in the relative abundance of Proteobacteria, a major phylum of gram-negative bacteria including Escherichia, Proteus, Salmonella, Klebsiella, and Morganella, was observed after bismuth quadruple therapy. These discrepant observations may be explained partially by different eradication regimens, drug doses and treatment duration lengths. In addition, other factors, such as dietary habit, previous antibiotic treatment history, and individual differences in the absorption rate for antibiotics, can affect the influence of eradication therapy on gut microbiota [25]. In our present study, we evaluated the food intake and baseline characteristics in each subjects and observed there was no obvious difference in the diet components and baseline characteristics. In terms of phylum level, probiotics supplementation did not obviously change the microbiome composition after eradication therapy.

There were important taxonomic changes at the genus level after treatment. BQT treatment at week 2 was associated with decreased abundance of Bacteroides, Faecalibacterium, Roseburia, Lachnospira, Phascolarctobacterium, Bifidobacterium, and Butyricimonas, most of which are known to have beneficial effects, such as producing the short chain fatty acid butyrate. On the contrary, there was an increase in relative abundances of some detrimental bacteria, such as Klebsiella, Streptococcus, Fusobacterium, Prevotella, and Morganella. Since amoxicillin and furazolidone have limited activity against these bacteria, it is likely that these detrimental bacteria may rapidly increase due to inhibition of other commensal bacteria. At week 2 after eradication, probiotics supplementation did not markedly alter the microbiota composition, suggesting the impact of antibiotics surpassed the protective effect of probiotics. With the prolonged eradication time, most of these beneficial and detrimental bacteria alterations were restored by week 6 and week 8, except for the persistent increase of Klebsiella in BQT group, implying that BQT therapy could lead to a persistent antibiotics resistance of Klebsiella. However, probiotics supplementation rapidly decreased the enrichment of Klebsilla, suggesting that the concomitant use of probiotics might be beneficial to reduce conditioned bacteria and antibiotics resistance. At week 2 and week 4, we observed a colonization of Enterococcus and Bacillus, the main components of the probiotics Medilac-S, while disappeared at week 6 and week 8, suggesting the colonization of probiotics closely depends on the supplementation duration. Most importantly, probiotics supplementation increased the beneficial bacteria such as Oscillospira and Lactobacillales at week 2 and week 4, which is reported to produce the short chain fatty acid butyrate, regulate host immune response and improve gastrointestinal symptoms [25, 27]. Furthermore, the probiotics supplementation reduced the abundance of Dialister, Sutterella, and Collinsella, mainly contributing to the digestive disorder, inflammation, abnormal lipids metabolism and various metabolic syndrome [28-31]. However, we did not observed much valuable difference at week 6 and week 8 between the two groups, probably because the probiotics supplementation was abolished in this period. 
The differences of functional profiles of gut microbiota after treatments were compared in antibiotic group and probiotic group. The proportion of pathways involved in starch degradation, glycolysis, and amino acid biosynthesis was decreased after treatment in both groups, whereas the proportion of pathways involved in the fatty acid oxidation and sucrose degradation increased. Various bacterial taxa such as Ruminococcus bromii and Bifidobacterium adolescentis are able to degrade starch [32], leading to an increase in specific fermentation end products, in particular butyrate, promoting epithelial integrity and immune homeostasis [33]. During the metabolism of glycolysis and amino acid biosynthesis, these compounds act as donors for sugar residues in glycosylation reactions that produce polysaccharides, which are important constituents of the cell wall $[34,35]$. Moreover, the increased incidence of sucrose metabolism and fatty acid oxidation after eradication therapy were closely associated with the obesity and metabolic syndrome[36, 37], which was consistent with some previous data indicating H. pylori eradication contributed to the metabolic parameters changes $[38,39]$. These intriguing findings suggest that $\mathrm{H}$. pylori eradication therapy might bring some potential detrimental effect through gut microbiota alterations, whether these changes are associated with significant clinical outcomes should be assessed in future studies. Under this circumstance, we further compared the changes of functional pathways between antibiotics group and probiotics group. Surprisingly, we only observed the distinct differences between the two groups at week 4. The proportion of pathways involved in the lipopolysaccharide biosynthesis, polymyxin resistance was increased only in the antibiotics group, while the metabolic pathways associated with metabolism of cofactors and vitamins were enriched in probiotics group. Enterococcus, the main components of probiotics, was positively correlated with thiamin diphosphate biosynthesis, tetrahydrofolate biosynthesis, and coenzyme A biosynthesis, while negative related to lipopolysaccharide biosynthesis, polymyxin resistance, suggesting probiotics supplementation might help to construct a beneficial profile of gut microbiota after eradication therapy.

We found that the variations of gut microbiome patterns are associated with several factors such as age, alcohol and BMI after eradication therapy. Several taxa can be identified to differentiate these clinical characteristics. In terms of age, we observed that Streptococcus and Dorea were significantly enriched in older patients after eradication therapy, while probiotics supplementation abolished this enrichment. Streptococcus is important conditioned pathogen causing inflammation and sepsis, and Dorea is the important acrobacter aerogenes contributing to the incidence of irritable bowel syndrome [40, 41]. For alcohol drinkers, Gemmiger and Collinsella were highly increased after eradication, which were associated with the abnormal lipid metabolism, obesity and metabolic syndrome [42, 43]. Moreover, for obese patients, Prevotella, Bilophila, Dialister, which have been reported to be enriched in obsess individuals [44], were also increased after eradication therapy. Intriguingly, the probiotics supplementation could effectively restore the adverse microbiome abundance in patients with the above characteristics, implying that $\mathrm{H}$. pylori eradication therapy might have the most impact on the aged, drinking and obese individuals, and probiotics supplementation would gain the maximum benefit for these subjects.

Nevertheless, there were some limitations to this study. First, although the strength of this study includes a large-scale, multicenter, randomized trial, longer duration of follow-up is needed to investigate the longterm effect of eradication therapy and probiotics in gut microbiota. Second, the changes in the species 
level and detailed function profiles after eradication therapy could not be assessed through 16S rRNA sequencing. Further whole genome shotgun sequencing would be needed.

\section{Conclusions}

Bismuth quadruple therapy leads to transient perturbation and changes in the gut microbiota immediately after therapy, most of these changes returned to pretreatment levels by 8 weeks. Although probiotics supplementation did not obviously change the microbiota diversity compared to the antibiotics group, probiotics supplementation during $\mathrm{H}$. pylori eradication might be beneficial to reduce conditioned bacteria and antibiotics resistance, which is closely dependent on the supplementation duration. Bismuth quadruple therapy might bring some potential detrimental effect through gut microbiota alterations, and probiotics supplementation changed the functional potential of gut microbiome, which is supposed to be beneficial to human health. Collectively, our results proposed that probiotics are beneficial for patients during $\mathrm{H}$. pylori eradication, especially for patients with older age, alcohol drinking, and obesity, which might obtain the maximum benefits.

\section{Declarations}

\section{Acknowledgements}

We thank all the individuals who participated in this study and donated samples. This study was supported by Science and Technology Promotion Project of AMU (2019XLC3022).

\section{Contributions}

SY, BT and JH contributed design of the study. HL, GZ, CH, CT, LC, ZH, GY, and LZ contributed to subject recruitment and sample collection. BT and SW completed DNA isolation. BT, LT, and EL completed analysis. BT and LT wrote the draft of the manuscript. SY and JH revised the manuscript. All authors read and approved the submitted version.

\section{Funding}

This study was supported by Science and Technology Promotion Project of AMU (2019XLC3022).

\section{Data availability statement}

All data relevant to the study are included in the article or uploaded as online supplementary information. 


\section{Ethics approval}

This trial was approved by the Institutional Review Board of each participating hospital.

\section{Consent for publication}

Not applicable.

\section{Competing interests}

The authors declare no competing financial interests.

\section{References}

1. Leja M, Grinberga-Derica I, Bilgilier C, Steininger C. Review: Epidemiology of H. pylori infection. Helicobacter. 2019;24 Suppl 1:e12635.

2. Waskito LA, Salama NR, Yamaoka Y. Pathogenesis of H. pylori infection. Helicobacter. 2018;23 Suppl 1:e12516.

3. Malfertheiner P, Megraud F, O'Morain CA, et al. Management of $H$. pylori infection-the Maastricht V/Florence Consensus Report. Gut. 2017;66(1):6-30.

4. Choi IJ, Kook MC, Kim Yl, et al. H. pylori Therapy for the Prevention of Metachronous Gastric Cancer. N Engl J Med. 2018;378(12):1085-1095.

5. O'Connor A, Liou JM, Gisbert JP, O'Morain C. Review: Treatment of H. pylori Infection 2019. Helicobacter. 2019;24 Suppl 1:e12640.

6. Liu WZ, Xie Y, Lu H, et al. Fifth Chinese National Consensus Report on the management of H. pylori infection. Helicobacter. 2018;23(2):e12475.

7. Blaser MJ. Antibiotic use and its consequences for the normal microbiome. Science. 2016;352(6285):544-545.

8. Hsu PI, Pan CY, Kao JY, et al. H. pylori eradication with bismuth quadruple therapy leads to dysbiosis of gut microbiota with an increased relative abundance of Proteobacteria and decreased relative abundances of Bacteroidetes and Actinobacteria. Helicobacter. 2018;23(4):e12498.

9. Yanagi $\mathrm{H}$, Tsuda $\mathrm{A}$, Matsushima $\mathrm{M}$, et al. Changes in the gut microbiota composition and the plasma ghrelin level in patients with $H$. pylori-infected patients with eradication therapy. BMJ Open Gastroenterol. 2017;4(1):e000182. Published 2017 Nov 26. 
10. Upala S, Jaruvongvanich V, Riangwiwat T, Jaruvongvanich S, Sanguankeo A. Association between $H$. pylori infection and metabolic syndrome: a systematic review and meta-analysis. J Dig Dis. 2016;17(7):433-440.

11. Upala S, Sanguankeo A, Saleem SA, Jaruvongvanich V. Effects of $H$. pylori eradication on insulin resistance and metabolic parameters: a systematic review and meta-analysis. Eur J Gastroenterol Hepatol. 2017;29(2):153-159.

12. Trifan A, Girleanu I, Cojocariu C, et al. Pseudomembranous colitis associated with a triple therapy for H. pylori eradication. World J Gastroenterol. 2013;19(42):7476-7479.

13. Modi SR, Collins JJ, Relman DA. Antibiotics and the gut microbiota. J Clin Invest. 2014;124(10):4212-4218.

14. Oh B, Kim BS, Kim JW, et al. The Effect of Probiotics on Gut Microbiota during the H. pylori Eradication: Randomized Controlled Trial. Helicobacter. 2016;21(3):165-174.

15. Liou JM, Chen CC, Chang CM, et al. Long-term changes of gut microbiota, antibiotic resistance, and metabolic parameters after $H$. pylori eradication: a multicentre, open-label, randomised trial. Lancet Infect Dis. 2019;19(10):1109-1120.

16. Chen L, Xu W, Lee A, et al. The impact of $H$. pylori infection, eradication therapy and probiotic supplementation on gut microenvironment homeostasis: An open-label, randomized clinical trial. EBioMedicine. 2018;35:87-96.

17. McNicholl AG, Molina-Infante J, Lucendo AJ, et al. Probiotic supplementation with Lactobacillus plantarum and Pediococcus acidilactici for H. pylori therapy: A randomized, double-blind, placebocontrolled trial. Helicobacter. 2018;23(5):e12529.

18. Shi X, Zhang J, Mo L, Shi J, Qin M, Huang X. Efficacy and safety of probiotics in eradicating H. pylori. A network meta-analysis. Medicine (Baltimore). 2019;98(15):e15180.

19. Callahan BJ, McMurdie PJ, Rosen MJ, Han AW, Johnson AJ, Holmes SP. DADA2: High-resolution sample inference from Illumina amplicon data. Nat Methods. 2016;13(7):581-583.

20. Weiss S, Xu ZZ, Peddada S, et al. Normalization and microbial differential abundance strategies depend upon data characteristics. Microbiome. 2017;5(1):27.

21. Quast C, Pruesse E, Yilmaz P, et al. The SILVA ribosomal RNA gene database project: improved data processing and web-based tools. Nucleic Acids Res. 2013;41(Database issue):D590-D596.

22. Gotoda T, Takano C, Kusano $C$, et al. Gut microbiome can be restored without adverse events after $H$. pylori eradication therapy in teenagers. Helicobacter. 2018;23(6):e12541.

23. Qin J, Li R, Raes J, et al. A human gut microbial gene catalogue established by metagenomic sequencing. Nature. 2010;464(7285):59-65.

24. Hsu PI, Pan CY, Kao JY, et al. Short-term and long-term impacts of $H$. pylori eradication with reverse hybrid therapy on the gut microbiota. J Gastroenterol Hepatol. 2019;34(11):1968-1976.

25. Astbury S, Atallah E, Vijay A, Aithal GP, Grove JI, Valdes AM. Lower gut microbiome diversity and higher abundance of proinflammatory genus Collinsella are associated with biopsy-proven 
nonalcoholic steatohepatitis. Gut Microbes. 2019;1-12.

26. Bartley A, Yang T, Arocha R, et al. Increased Abundance of Lactobacillales in the Colon of BetaAdrenergic Receptor Knock Out Mouse Is Associated With Increased Gut Bacterial Production of Short Chain Fatty Acids and Reduced IL17 Expression in Circulating CD4 ${ }^{+}$Immune Cells. Front Physiol. 2018;9:1593.

27. Jiao N, Baker SS, Nugent CA, et al. Gut microbiome may contribute to insulin resistance and systemic inflammation in obese rodents: a meta-analysis. Physiol Genomics. 2018;50(4):244-254.

28. Tito RY, Cypers H, Joossens M, et al. Brief Report: Dialister as a Microbial Marker of Disease Activity in Spondyloarthritis. Arthritis Rheumatol. 2017;69(1):114-121.

29. Del Chierico F, Nobili V, Vernocchi P, et al. Gut microbiota profiling of pediatric nonalcoholic fatty liver disease and obese patients unveiled by an integrated meta-omics-based approach. Hepatology. 2017;65(2):451-464.

30. Gomez-Arango LF, Barrett HL, Wilkinson SA, et al. Low dietary fiber intake increases Collinsella abundance in the gut microbiota of overweight and obese pregnant women. Gut Microbes. 2018;9(3):189-201.

31. Astbury S, Atallah E, Vijay A, Aithal GP, Grove JI, Valdes AM. Lower gut microbiome diversity and higher abundance of proinflammatory genus Collinsella are associated with biopsy-proven nonalcoholic steatohepatitis [published online ahead of print, 2019 Nov 7]. Gut Microbes. 2019;1-12.

32. Ze X, Duncan SH, Louis P, Flint HJ. Ruminococcus bromii is a keystone species for the degradation of resistant starch in the human colon. ISME J. 2012;6(8):1535-1543.

33. Smith PM, Howitt MR, Panikov N, et al. The microbial metabolites, short-chain fatty acids, regulate colonic Treg cell homeostasis. Science. 2013;341(6145):569-573.

34. Agus A, Planchais J, Sokol H. Gut Microbiota Regulation of Tryptophan Metabolism in Health and Disease. Cell Host Microbe. 2018;23(6):716-724.

35. Del Chierico F, Abbatini F, Russo A, et al. Gut Microbiota Markers in Obese Adolescent and Adult Patients: Age-Dependent Differential Patterns. Front Microbiol. 2018;9:1210.

36. Parks BW, Nam E, Org E, et al. Genetic control of obesity and gut microbiota composition in response to high-fat, high-sucrose diet in mice. Cell Metab. 2013;17(1):141-152.

37. Lee J, Choi J, Selen Alpergin ES, et al. Loss of Hepatic Mitochondrial Long-Chain Fatty Acid Oxidation Confers Resistance to Diet-Induced Obesity and Glucose Intolerance. Cell Rep. 2017;20(3):655-667.

38. Polyzos SA, Kountouras J, Zavos C, Deretzi G. The association between $H$. pylori infection and insulin resistance: a systematic review. Helicobacter. 2011;16(2):79-88.

39. Kountouras J, Polyzos SA, Doulberis M, et al. Potential impact of $H$. pylori-related metabolic syndrome on upper and lower gastrointestinal tract oncogenesis. Metabolism. 2018;87:18-24.

40. Jans C, Meile L, Lacroix C, Stevens MJ. Genomics, evolution, and molecular epidemiology of the Streptococcus bovis/Streptococcus equinus complex (SBSEC). Infect Genet Evol. 2015;33:419-436. 
41. Strati F, Cavalieri D, Albanese D, et al. New evidences on the altered gut microbiota in autism spectrum disorders. Microbiome. 2017;5(1):24.

42. Patrone V, Vajana E, Minuti A, et al. Postoperative Changes in Fecal Bacterial Communities and Fermentation Products in Obese Patients Undergoing Bilio-Intestinal Bypass. Front Microbiol. 2016;7:200.

43. Nirmalkar K, Murugesan S, Pizano-Zárate ML, et al. Gut Microbiota and Endothelial Dysfunction Markers in Obese Mexican Children and Adolescents. Nutrients. 2018;10(12):2009.

44. Thingholm LB, Rühlemann MC, Koch M, et al. Obese Individuals with and without Type 2 Diabetes Show Different Gut Microbial Functional Capacity and Composition. Cell Host Microbe. 2019;26(2):252-264.e10.

\section{Figures}




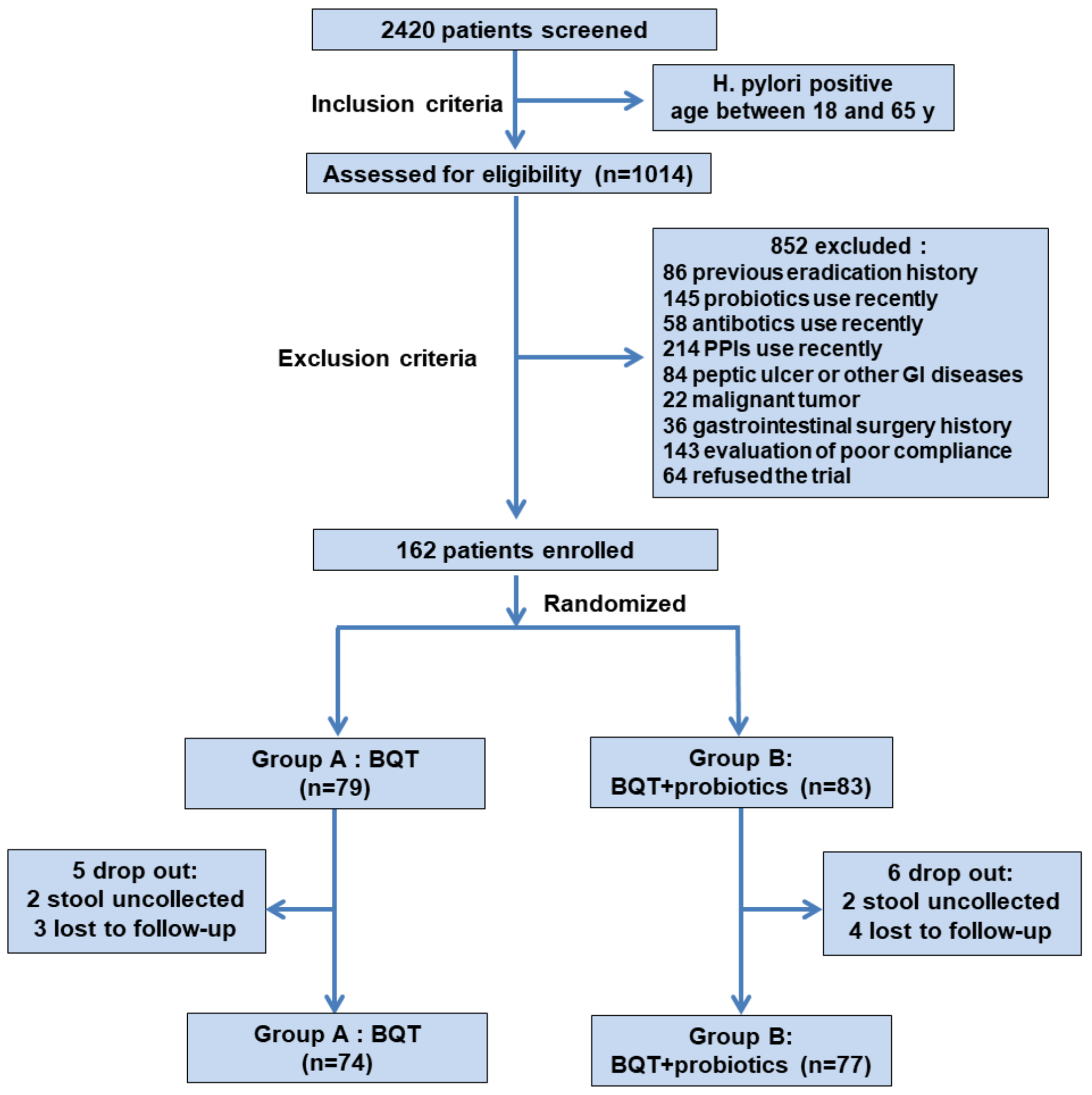

Figure 1

Flowchart of patient enrollment. BQT, bismuth quadruple therapy. 

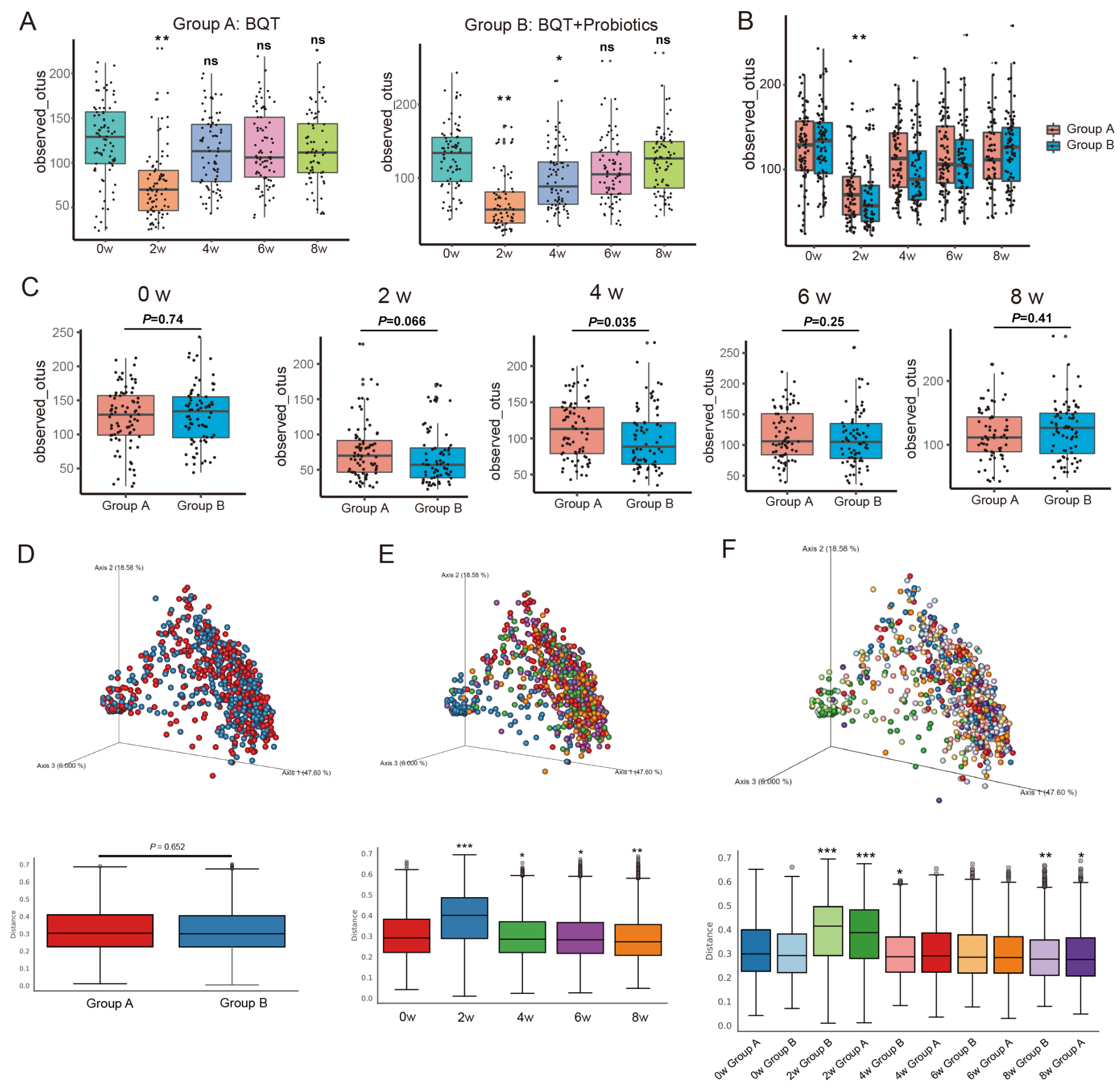

Figure 2

Comparison of the diversity between the two groups after eradication therapy. Alpha diversity (A-C) indices alterations were compared between Group A and Group B before treatment, at week 2, at week 4, at week 6 , and at week 8 . Beta diversity (D-F; principal coordinate analysis) was compared between the two groups at different time periods. 
A

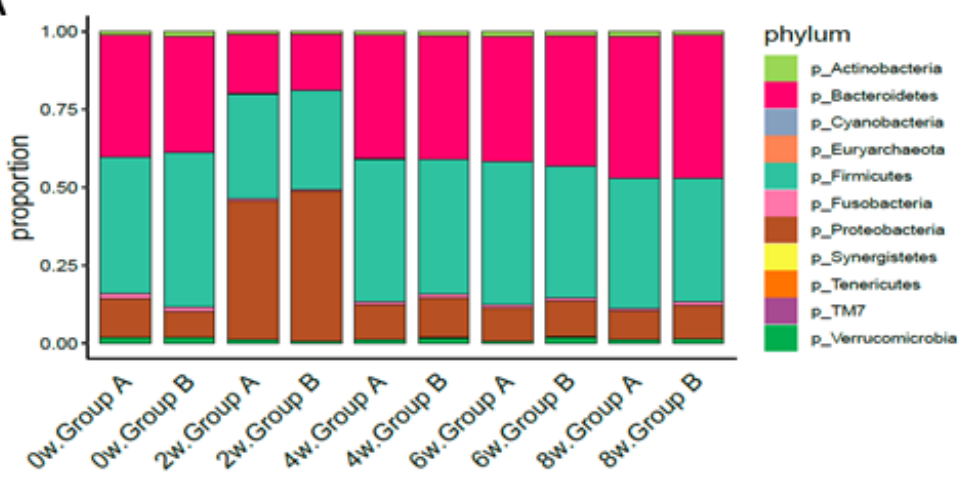

C

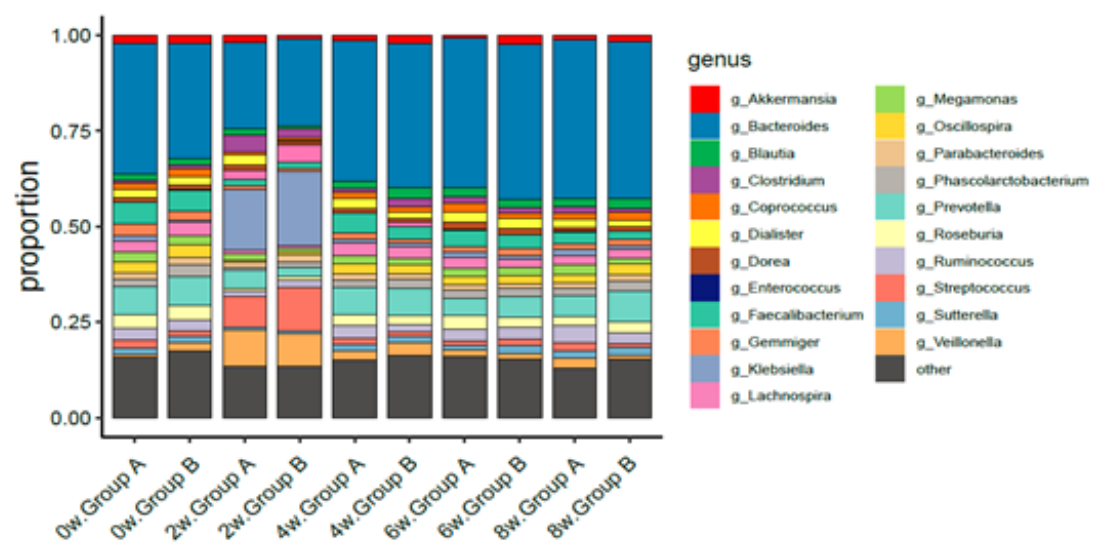

B

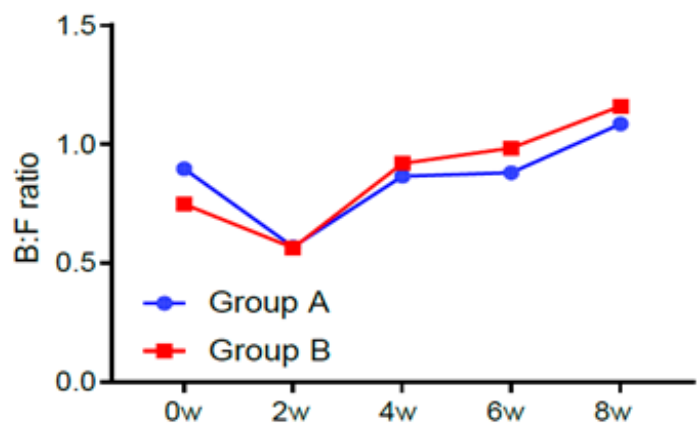

D

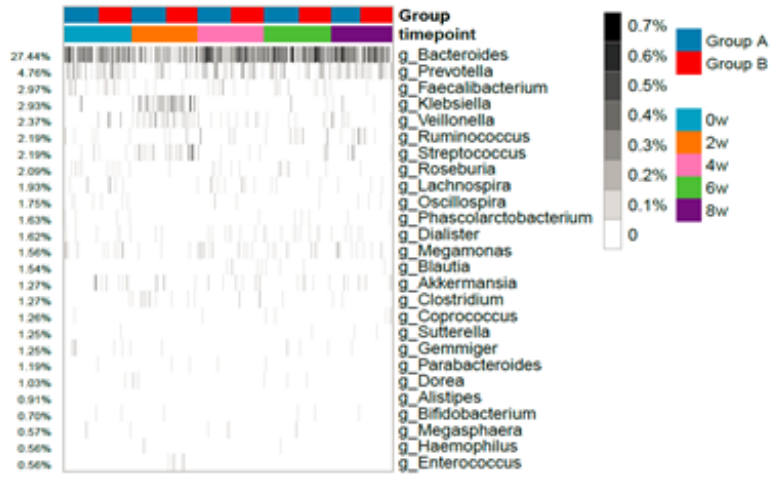

Figure 3

Taxonomic differences in gut microbiota composition between the two groups at different period. (A) Profiles of the relative abundance per group at phylum level. (B) Bacteroidetes:Firmicutes (B:F) ratios in two groups in different time points. (C) Profiles of the relative abundance per group at genus level. (D) Heat map showing the significantly changed taxa in group A and group B in different time points after eradication. 
A

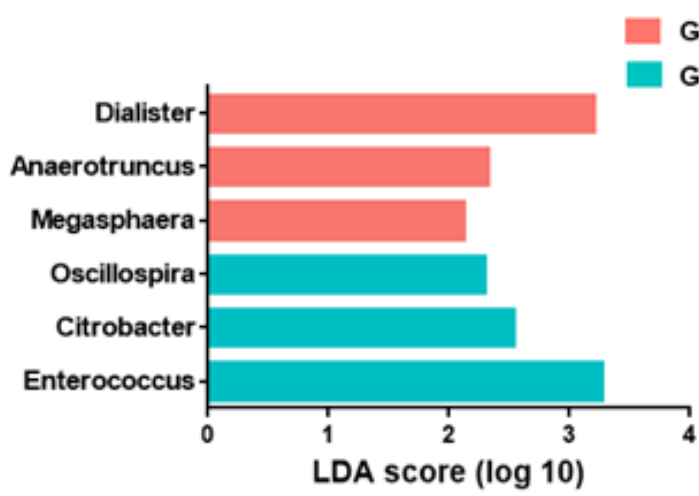

C

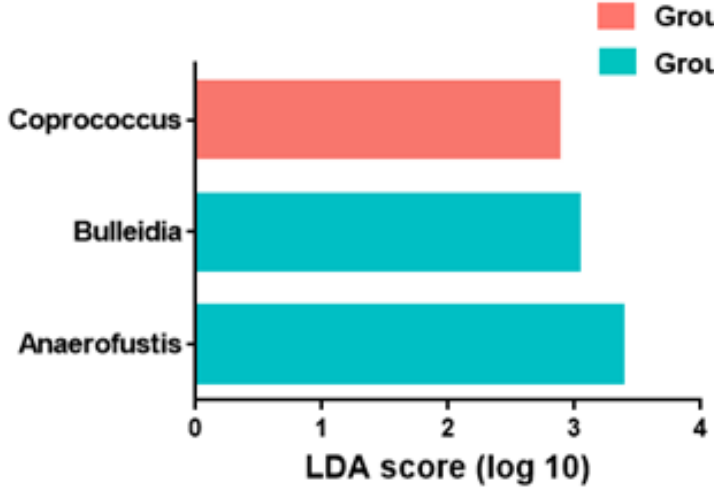

B

Group A_2w

Group B_2w

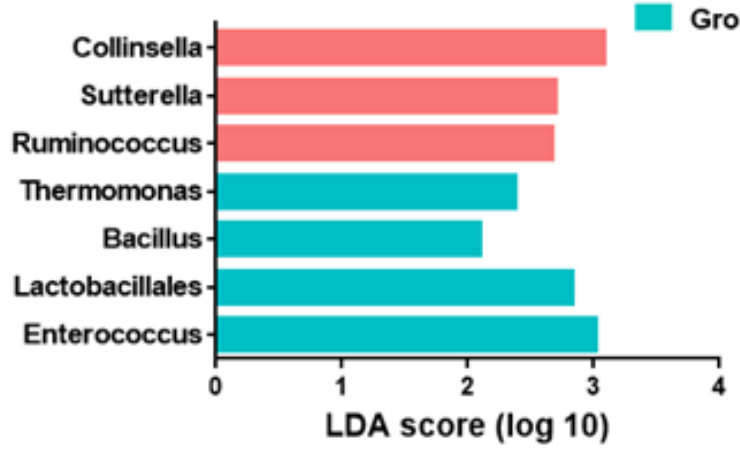

D

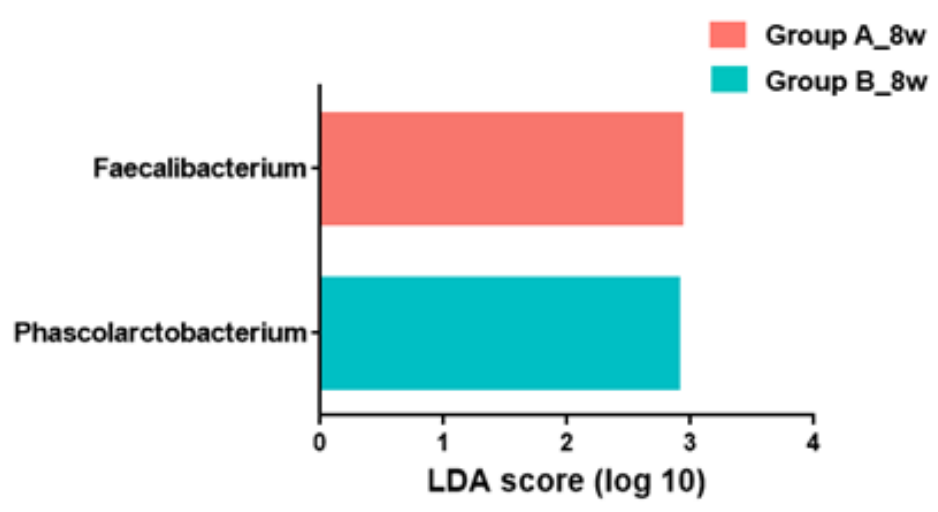

\section{Figure 4}

Comparison of the distinct gut bacterial taxa between group $A$ and group $B$ after eradication therapy. (AD) Bacterial taxa that are compared between group A and group B at week 2 (A), week 4 (B), week 6 (C), week 8 (D) were shown by LDA score at genus levels. Taxa with $P<0.01$ and LDA score $>2$ were considered significant and are shown here. 
A

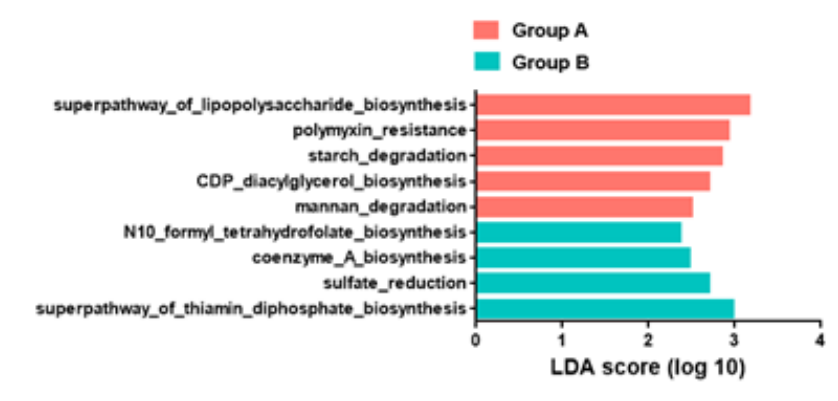

D

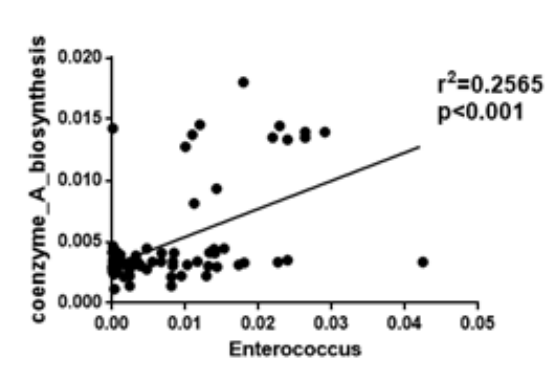

G

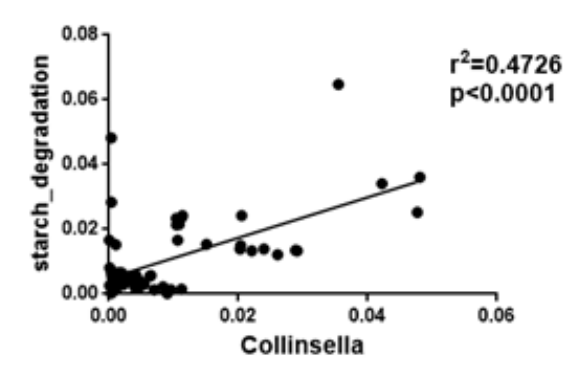

B

E

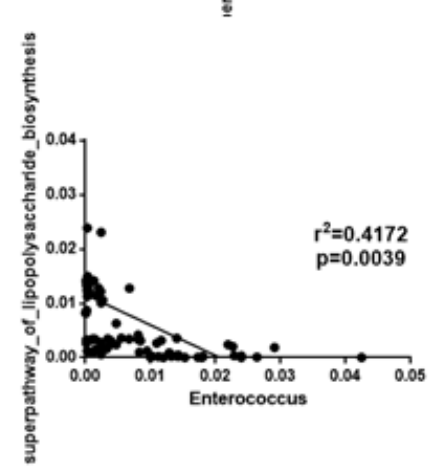

H

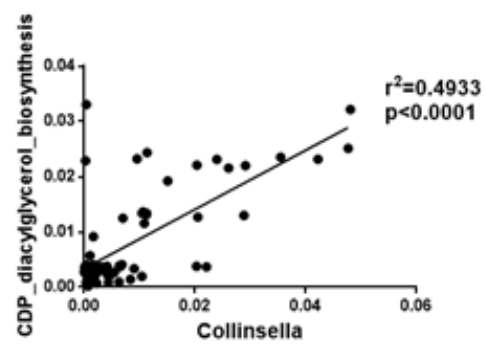

C

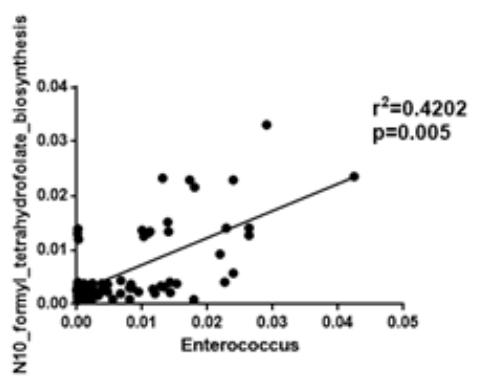

F
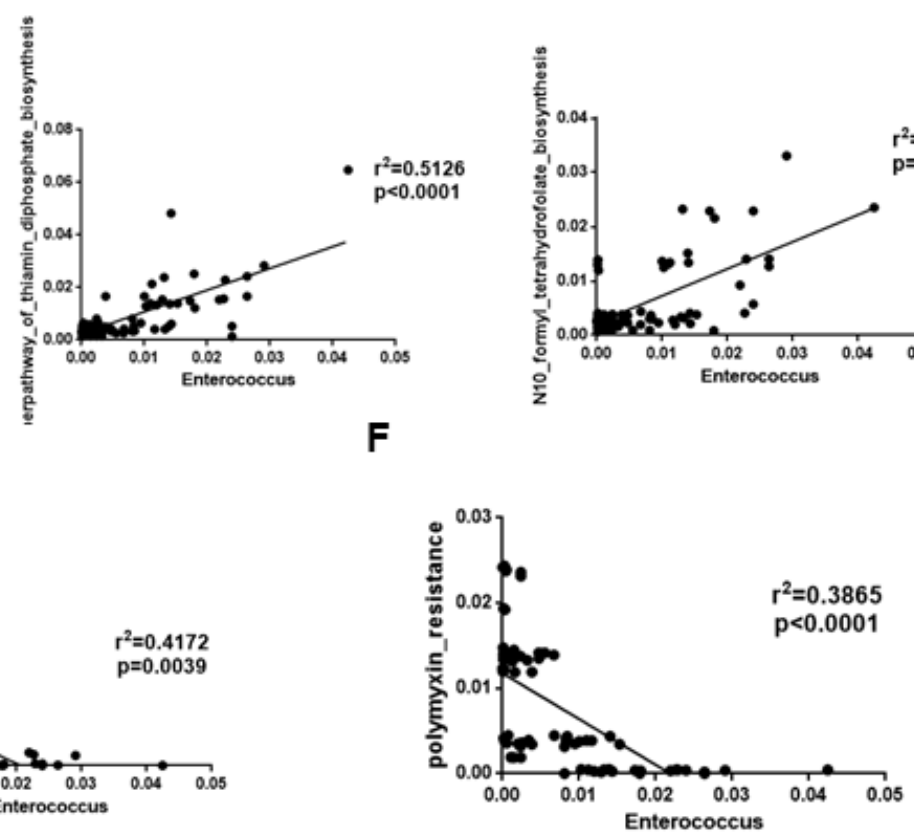

I

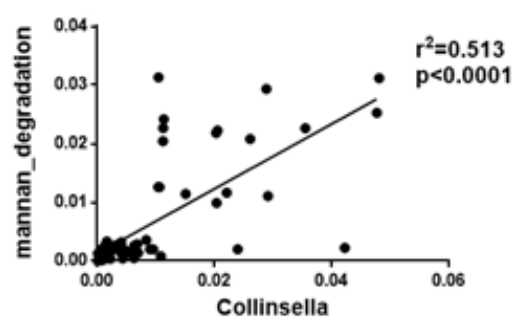

Figure 5

The distinct predicted metabolic pathways between the two groups. (A) The significantly different predicted metabolic pathways between group A and group B were shown by LDA scores analyzed by LEfSe. (B-F) The association of the relative abundance of Enterococcus with various pathways. (G-I) The association of the relative abundance of Collinsella with various pathways. 
A
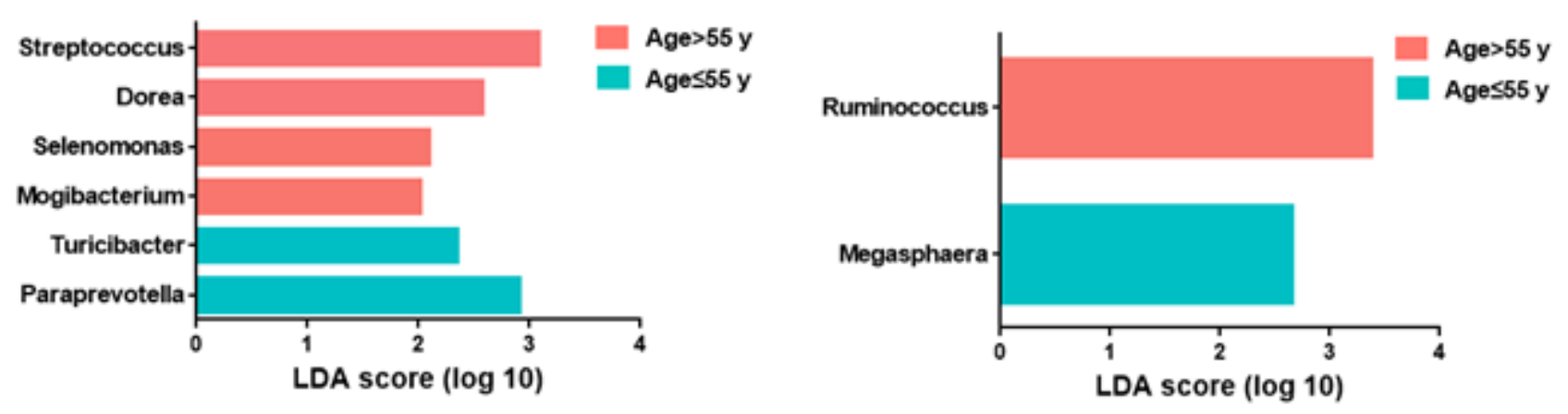

B
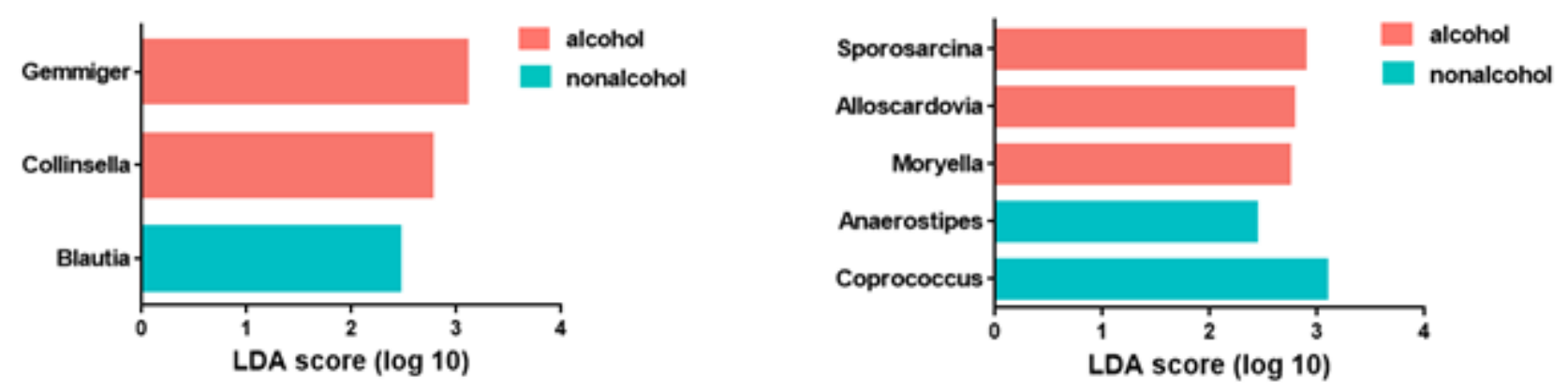

C
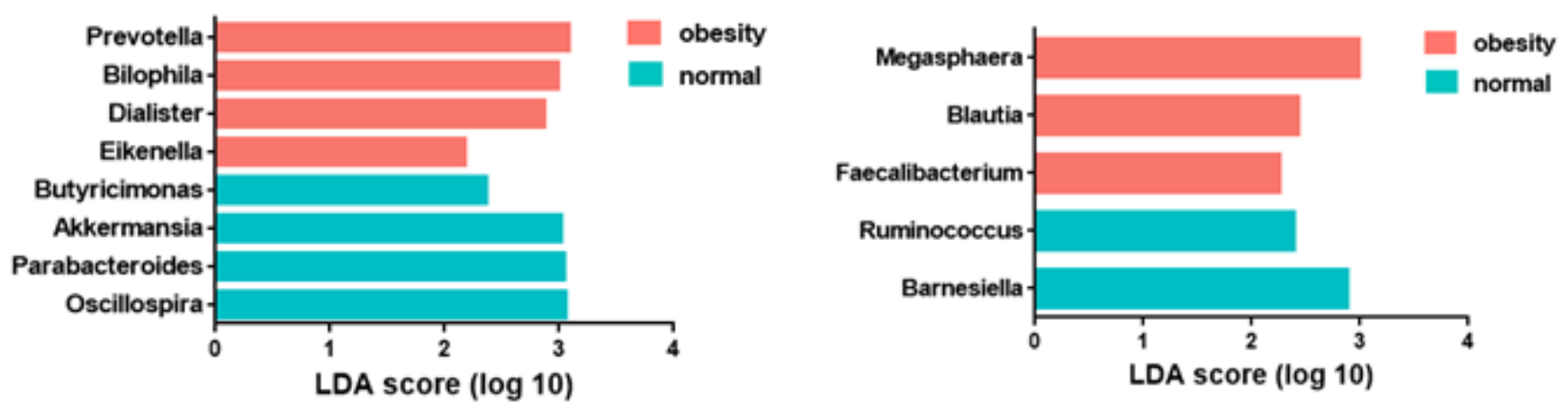

\section{Figure 6}

Linear discriminant analysis (LDA) of specific gut bacteria genera enriched in the two groups with different clinical features. (A) Bacterial taxa that are enriched in group $A$ and group $B$ with different ages; (B) Bacterial taxa that are enriched in group $A$ and group $B$ with alcohol drinking or not; (C) Bacterial taxa that are enriched in group $A$ and group $B$ with obesity or not.

\section{Supplementary Files}

This is a list of supplementary files associated with this preprint. Click to download.

- Supplementarymaterials.docx 